\title{
Using phases to determine the space group
}

Many of the structures that were Marshed involved an incorrect choice of space group. Traditionally, the choice of space group involved the determination of the Laue group, an analysis of potentially systematic absent reflections and possibly statistical tests to detect an inversion center. The program SHELXT[1] still requires the Laue group but solves the structure by dual-space direct methods using reflection data expanded to triclinic and then uses the resulting phases to determine the space group. This approach will be examined critically and several recent improvements in SHELXT described.

[1] G. M. Sheldrick, Acta Cryst. 2015, A71, 3-8.

George Sheldrick

Goettingen University

gsheldr@uni-goettingen.de 